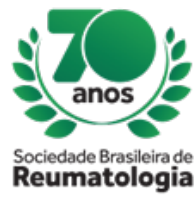

\title{
SECONDARY SJOGREN'S SYNDROME TO SYSTEMIC LUPUS ERYTHEMATOSUS, SYSTEMIC SCLEROSIS AND RHEUMATOID ARTHRITIS: EVALUATION OF CLINICAL, LABORATORY AND HISTOPATHOLOGICAL PROFILE OF PATIENTS REFERRED TO A TERTIARY HOSPITAL IN BRAZIL. A 5-YEAR FOLLOW-UP.
}

Saulo Musse Dib (HCFMRP-USP, Ribeirão Preto, SP, Brasil), Letícia Pastorelli Bonjorno (HCFMRP-USP, Ribeirão Preto, SP, Brasil), Marcela Cavichioli Giannini (HCFMRP-USP, Ribeirão Preto, SP, Brasil), Marcos Afonso Cardozo Jacinto (HCFMRP-USP, Ribeirão Preto, SP, Brasil), Maria Eugenia Teixeira Farias (HCFMRP-USP, Ribeirão Preto, SP, Brasil), Natália Brasil Amaral (HCFMRP-USP, Ribeirão Preto, SP, Brasil), Samara Libich Gusmão Gigante (HCFMRP-USP, Ribeirão Preto, SP, Brasil), Pamella Indira da Silva Oliveira Menezes (HCFMRP-USP, Ribeirão Preto, SP, Brasil), Alfredo Ribeiro Silva (HCFMRP-USP, Ribeirão Preto,

SP, Brasil), Flavio Calil Petean (HCFMRP-USP, Ribeirão Preto, SP, Brasil), Eduardo Melani Rocha (HCFMRP-USP, Ribeirão Preto, SP, Brasil), Paulo Louzada-Jr (HCFMRP-USP, Ribeirão Preto, SP, Brasil), Fabiola Reis Oliveira (HCFMRP-USP, Ribeirão Preto, SP, Brasil)

\section{BACKGROUND}

Sjögren's syndrome (SS) is a chronic inflammatory autoimmune disease, characterized by the involvement of exocrine glands, xerophthalmia and xerostomia. It may occur as primary SS or combined with another rheumatic immunomediated disease, secondary Sjögren's syndrome (SSS), such as Systemic Lupus Erythematosus (SLE), Systemic Sclerosis (SE) or Rheumatoid Arthritis (RA). This association promotes distinct clinical variants, as a less severe presentation in SLE-sSS and more severe RA-sSS, peculiarities in the autoantibody profile and in minor salivar glands histology, as higher fibrosis rates in SE-sSS. Secundary SS is underdiagnosed. Available data reveal an occurrence of 6.5-19\% among SLE patients (with less glomerulonephritis and central nervous system involvement). RA-sSS occurs in $26-31 \%$ of RA cases (with increased risk of non-Hodgkin's lymphoma and increased mortality). SE-sSS appearances with an occurrence of $14-20.5 \%$.

\section{MATERIALS AND METHODS}

Data collected during a 5-year follow-up, from SS patient's records, in a tertiary hospital. We describe 106 sSS subjects distributed into groups: SLE $(n=58)$, SE $(n=14), R A(n=34)$, according to the AECG-2002 classification criteria. Patients were submitted to clinical and ocular evaluation, including Schirmer's test, tear film break-up time, fluorescein Staining Score and sialometry. We reported the frequency of autoantibodies and characteristics of minor salivar glands histology. The descriptive and comparative statistics frequency of positive results in each group were analyzed.

\section{RESULTS}

SLE-sSS patients were younger than RA-SSS $(p<0.0001)$. The positivity of dry eyes and dry mouth symptoms was similar among the groups. The percentage of positivity SSa in SLE-sSS was higher than in SE-sSS and RA-sSS, respectively $79.3 \%, 28.6 \%$ and $26.5 \%(p<0.0001)$. RF titers were higher in RA-sSS compared to SLE-sSS, as expected ( $p<0.0001$ ). RA-sSS and SE-sSS had focus score $=1$ in $61.8 \%$ and $64.3 \%$, respectively, higher than $\mathrm{SLE}-\mathrm{sSS}, 36.2 \%(\mathrm{p}=0.0018)$. There was no statistic difference in functional tests results between groups.

\section{CONCLUSION}

Our results relate to the limited data in the literature. Dry symptoms and functional tests (sialometry and Schirmer-I) were not able to discriminate patients, reinforcing the importance of salivary gland biopsy. Metacentric studies with larger casuistry are necessary, particularly to recognize possible differences in glandular lesion in SLE-sSS and SE-sSS. The heterogeneity of sSS, depending on the underlying disease, should be considered when selecting cases for studies. 